\title{
$e^{+} e^{-}$hadronic cross section and the muon $g-2$
}

\author{
Concetta Cartaro ${ }^{1, a}$ \\ ${ }^{1}$ On behalf of the BABAR Collaboration, \\ SLAC National Accelerator Laboratory, 2575 Sand Hill Road - Menlo Park, 94025 CA - USA
}

\begin{abstract}
.
After a brief introduction to the muon anomalous magnetic moment, the current status of the $(g-2)_{\mu}$ measurement is given. The three standard deviations of discrepancy between $a_{\mu}$ calculations and the direct measurement by the E821 experiment at the Brookhaven National Laboratory (USA) calls for more precise measurements of the $e^{+} e^{-}$hadronic cross section. This article contains a not inclusive review of the main results and the state of the art on the hadronic cross section measurements from $B A B A R$ and other experiments.
\end{abstract}

\section{Introduction to the $(g-2)_{\mu}$ anomaly}

The magnetic moment of the muon (or of any lepton, provided to use the right mass) is defined as:

$$
\vec{\mu}=g \frac{e \hbar}{2 m_{\mu} c} \vec{s}
$$

where $\vec{s}$ is the muon spin, $m_{\mu}$ is its mass, and $e$ is the signed electric charge.

In the Dirac theory $g=2$ and the anomalous magnetic moment of the muon, defined as

$$
a_{\mu}=(g-2) / 2,
$$

is exactly $a_{\mu}=0$. However in quantum field theory $g \neq 2$ because of higher order corrections in QED and other interactions. In the Standard Model (SM) the anomalous muon magnetic moment is split into different contributions:

$$
a_{\mu}^{S M}=a_{\mu}^{Q E D}+a_{\mu}^{\text {weak }}+a_{\mu}^{\text {had }}
$$

The $a_{\mu}^{Q E D}$ [1] and $a_{\mu}^{\text {weak }}$ [2] terms are very well known with little uncertainty. The last term, $a_{\mu}^{\text {had }}$, is the main source of theoretical uncertainty. The major contributions come from the lowest order (LO) hadronic vacuum polarization (see Feynman diagram in Figure 1), some higher order vacuum polarization (photon and hadronic loops in the photon propagator), and a light-by-light contribution where the hadronic loop has four photon propagators connected to the QED diagram.

The LO contribution of the hadronic vacuum polarization to $a_{\mu}^{\text {had }}$ can be calculated using the dispersion integral:

\footnotetext{
a e-mail: cartaro@slac.stanford.edu
} 


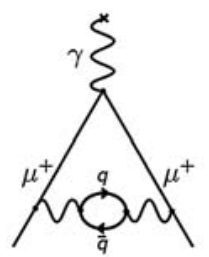

Figure 1. Lowest order Feynman diagram of vacuum polarization contributing to the muon anomaly.

Table 1. Main contributions to $a_{\mu}$ and their uncertainty. In the last line the E821 experimental result and the expectation in the Standard Model (SM) are compared [5].

\begin{tabular}{lrr}
\hline Source & Value $\left(10^{-10}\right)$ & Uncertainty $\left(10^{-10}\right)$ \\
\hline QED & 11658471.809 & 0.015 \\
Weak & 15.4 & 0.2 \\
Hadronic, LO & 692.3 & 4.2 \\
BNL E821 & 11659208.9 & 6.4 \\
\hline (BNL E821) - (SM Theory) & 28.7 & 8.0 \\
\hline
\end{tabular}

$$
a_{\mu}^{h a d, L O}=\frac{\alpha^{2}(0)}{3 \pi^{2}} \int_{4 m_{\pi}^{2}}^{\infty} d s \frac{K(s)}{s} R(s)
$$

where $\alpha(s)$ is the running electromagnetic coupling constant, $R(s)$ is the ratio of the bare (no vacuum polarization) $e^{+} e^{-}$hadronic cross section and the point like muon-pair cross section. $K(s)$ is the QED kernel function [3] and decreases monotonically with $s$. This makes the low energy contributions to the hadronic cross section particularly important. In particular more than $90 \%$ of the contribution to $a_{\mu}^{\text {had }}$ is given by center of mass energies below $1.8 \mathrm{GeV}$, dominated by the two pion final state from the $\rho(770)$ resonance.

The most precise experimental measurement of $a_{\mu}^{\text {had }}$ has been achieved at the Brookhaven National Laboratory by the E821 experiment [4] and it differs from the theoretical prediction in the Standard Model of $a_{\mu}^{\text {had }}$ by 3.6 standard deviations.

Table 1 summarizes the main contributions to the muon magnetic moment anomaly and their uncertainty. The data used in the table are from reference [5].

\section{Experimental input for $a_{\mu}^{\text {had }}$ and the ISR method}

The traditional way to measure the hadronic $e^{+} e^{-}$cross section as function of energy is to use data from $e^{+} e^{-}$scan experiments, collected by changing the beam energy in all the accessible energy range. Very large data sets exist in the energy region below $1.4 \mathrm{GeV}$ collected by SND and CMD-2 experiments at VEPP-2M in Novosibirsk (Russia). Above this value very little data exist, collected mainly by DM1 and DM2 experiments at DCI collider in Orsay (France). The Initial State Radiation (ISR) method, used by BABAR at the PEP-II accelerator [6] in Menlo Park (USA) and other experiments like KLOE at DAФNE in Frascati (Italy) or, more recently, BESIII at BEPCII in Beijing (China), takes advantage of the high precision allowed by the large data sets and the high detector performances in an energy region previously unreachable. 
In $e^{+} e^{-} \rightarrow X$ annihilation processes at accelerators, the center of mass energy is given by $\sqrt{s}=$ $2 E_{b}^{*}$, where $E_{b}^{*}$ is the beam energy in the center of mass. However the colliding electrons can emit photon(s) in the initial state effectively lowering the center of mass energy, giving continuous access to energies from the final state production threshold up to $2 E_{b}^{*}$.

\subsection{The ISR method at BABAR}

The BABAR experiment is characterized by asymmetric $e^{+}$and $e^{-}$energy beams $(3.1 \mathrm{GeV}$ and $9 \mathrm{GeV}$, respectively) generating a boost of $\beta \gamma=0.56$ of the center of mass in the laboratory system. The data are collected at the production energy of the $\Upsilon(4 S)$ resonance at $10.58 \mathrm{GeV}$ (on peak) or $40 \mathrm{MeV}$ below it (off peak).

At $B A B A R$, the study of the ISR production of light hadrons is performed requiring the detection of the ISR photon with an energy above $3 \mathrm{GeV}$ in the $e^{+} e^{-}$center of mass system. Less then about $15 \%$ of the ISR photons are emitted within the geometrical acceptance of the electromagnetic calorimeter. On the other hand, the loss of efficiency is compensated by a very good background rejection.

The topology of the events is such that the ISR photon is back-to-back with the hadron system which allows to reach, thanks to the boost, detection efficiencies greater then zero even at the production threshold of the hadronic system. The center of mass energy after the emission of the ISR photon is equivalent to the invariant mass of the final hadronic system, which is measured with a resolution of a few $\mathrm{MeV} / \mathrm{c}^{2}$.

The ISR method gives continuous access to the energy range from threshold up to $5 \mathrm{GeV}$ using the same experimental setup and data taking conditions, providing common, consistent systematic uncertainties.

The BABAR experiment has a very large program for ISR physics including light meson spectroscopy. Some of the most relevant and recent results are briefly described in the following sections.

\subsection{The $e^{+} e^{-} \rightarrow \pi^{+} \pi^{-}$hadronic cross section}

The $e^{+} e^{-} \rightarrow \pi^{+} \pi^{-}(\gamma)$ cross section dominates in the energy region $\mathrm{E}<1 \mathrm{GeV}$. This very precise analysis was performed in 2009 on $232 \mathrm{fb}^{-1}$ by BABAR [7][8]. The final states $\pi^{+} \pi^{-}(\gamma)$ and $\mu^{+} \mu^{-}(\gamma)$ are measured, where the $\gamma$ accompanying the charged pair is a final state radiation (FSR) photon. Extracting the $\pi^{+} \pi^{-}$cross sections from the hadron to muon ratio greatly reduces the systematic uncertainties. The main background contamination comes from other ISR events with one or more $\pi^{0}$. Events like $e^{+} e^{-} \rightarrow \gamma \gamma$ with photon conversion are irreducible backgrounds and are taken into account. The level of systematic uncertainty is $0.5 \%$ around the $\rho(770)$ resonance. The measured cross section is shown Figure 2. In Figure 3 are shown, on the left, the comparison between the measured cross section above $1 \mathrm{GeV}$ at $B A B A R, C M D-2$, and DM2 in their respective energy ranges, and on the right, the comparison between the measured pion form factor (defined as the ratio of the cross section without FSR, divided by the lowest-order cross section for point-like spin 0 charged particles), in the region below $1 \mathrm{GeV}$, at $B A B A R$ and KLOE.

\subsection{The $e^{+} e^{-} \rightarrow K^{+} K^{-}$hadronic cross section}

The $e^{+} e^{-} \rightarrow K^{+} K^{-}(\gamma)$ hadronic cross section dominates in the energy range $1 \mathrm{GeV}<\mathrm{E}<2 \mathrm{GeV}$ and it also is, in this energy range, the single largest contributor to the uncertainty in the dispersion integral evaluation, mainly due to the presence of the $\phi(1020)$ resonance. This analysis [9], performed on the same data set used for the study of the $\pi^{+} \pi^{-}$final state, aims at keeping the systematic uncertainties 


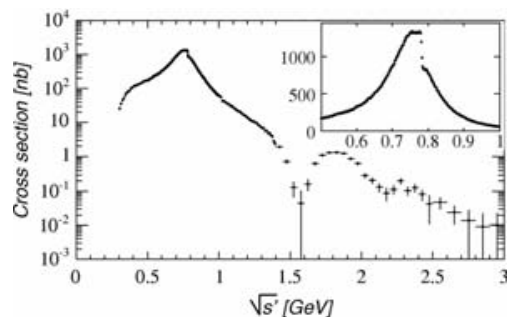

Figure 2. The $e^{+} e^{-} \rightarrow \pi^{+} \pi^{-}$hadronic cross section at BABAR. The spectrum is dominated by the $\rho(770)$ resonance and interference between $\rho$ and $\omega$ resonances can be observed in the peak region (the inset is a magnification of the peak). The other features at $1.6 \mathrm{GeV}$ and $2.2 \mathrm{GeV}$ are interpreted as the interference between $\rho^{\prime}$ and $\rho^{\prime \prime}$ resonances, and an even higher mass $\rho$ state, respectively.
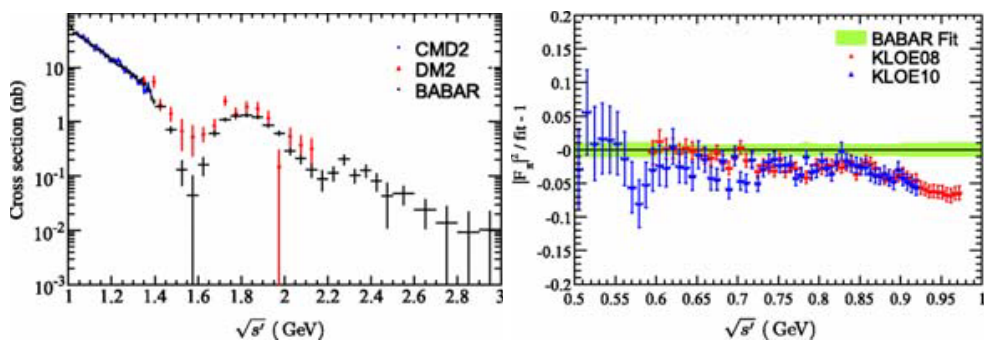

Figure 3. The $e^{+} e^{-} \rightarrow \pi^{+} \pi^{-}$hadronic cross section at BABAR and other experiments. On the left the comparison between $B A B A R, C M D-2$, and DM2 are shown. The systematic uncertainties range from the $0.5 \%$ of $B A B A R$, to the $0.8 \%$ of KLOE and CMD-2. On the right plot, in the region below $1 \mathrm{GeV}$, the comparison between the pion form factors measured at BABAR and KLOE is shown. A slight deviation between the two experiments can be observed above $\rho$ peak region.

under the $1 \%$ level and a precise measurement of the $\phi$ parameters. The contribution to the anomalous magnetic moment of the muon using the dispersion integral from the $K^{+} K^{-}$threshold up to $1.8 \mathrm{GeV}$ is: $a_{\mu}^{K K, L O}=\left(22.93 \pm 0.18_{\text {stat }} \pm 0.22_{\text {syst }} \pm 0.03_{V P}\right) \times 10^{-10}$, where the uncertainties are, in the order, statistical, systematic, and from the $\phi$ parameters used in the vacuum polarization correction. The total precision is $1.2 \%$ with the systematic uncertainty reaching the $0.8 \%$ level at the $\phi$ peak. The measured $\mathrm{K}^{+} \mathrm{K}^{-}$cross section is reported in Figure 4.

\subsection{The $e^{+} e^{-} \rightarrow K_{S}^{0} K_{L}^{0}$ hadronic cross section}

The $e^{+} e^{-} \rightarrow K_{S}^{0} K_{L}^{0}$ hadronic cross section gives access to the $1 \mathrm{GeV}<\mathrm{E}<2.2 \mathrm{GeV}$ energy range. The cross section is dominated by the $\phi(1020)$ peak where it is measured $(1409 \pm 55) \mathrm{nb}$. The systematic uncertainty at the peak is $2.9 \%, 10 \%$ above $0.5 \mathrm{nb}$, and $30 \%$ below $0.5 \mathrm{nb}$, dominated by backgound subtraction. This BABAR analysis is described in reference [10] and is performed on the final BABAR data set of $468 \mathrm{fb}^{-1}$ including both the on peak and the off peak data.

\subsection{The $e^{+} e^{-} \rightarrow K_{S}^{0} K_{L}^{0} \pi^{+} \pi^{-}, K_{S}^{0} K_{S}^{0} \pi^{+} \pi^{-}$, and $K_{S}^{0} K_{S}^{0} K^{+} K^{-}$hadronic cross sections}

The same BABAR paper in reference [10] also reports the first observation of the $J / \psi \rightarrow K_{S}^{0} K_{L}^{0} \pi^{+} \pi^{-}$, $K_{S}^{0} K_{S}^{0} \pi^{+} \pi^{-}$, and $K_{S}^{0} K_{S}^{0} K^{+} K^{-}$hadronic modes, previously evaluated only through isospin relations. 


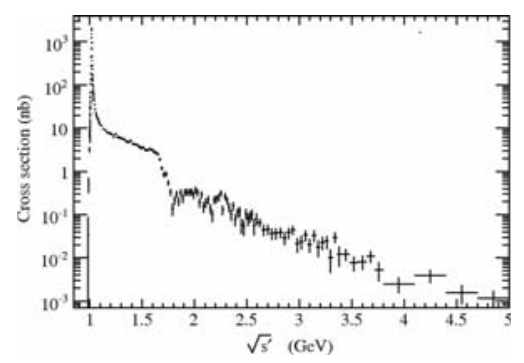

Figure 4. The $e^{+} e^{-} \rightarrow K^{+} K^{-}$hadronic bare cross section (including FSR) at BABAR. The $J / \psi$ and $\psi(2 S)$ resonances to $K^{+} K^{-}$have been subtracted. The systematic uncertainty at the $\phi$ peak is $0.8 \%$.
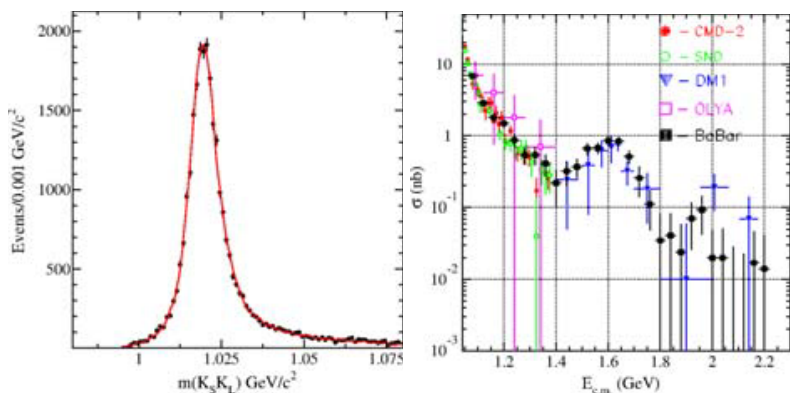

Figure 5. The $e^{+} e^{-} \rightarrow K_{S}^{0} K_{L}^{0}$ hadronic cross section at BABAR. In the left plot the $K_{S}^{0} K_{L}^{0}$ in the $\Phi(1020)$ is shown. The right plot contains the comparison of the $K_{S}^{0} K_{L}^{0}$ cross section from the data of all relevant experiments.

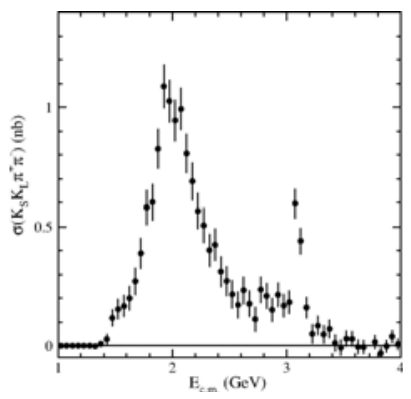

Figure 6. The $e^{+} e^{-} \rightarrow K_{S}^{0} K_{L}^{0} \pi^{+} \pi^{-}$hadronic cross section at $B A B A R$. The peak at $3.1 \mathrm{GeV}$ is the $J / \psi$ resonance.

The measured branching fraction for the first of these modes is $B\left(J / \psi \rightarrow K_{S}^{0} K_{L}^{0} \pi^{+} \pi^{-}\right)=(3.7 \pm$ $0.6 \pm 0.4) \times 10^{-3}$. The systematic uncertainty is dominated by the background subtraction, the $K^{*+} K^{*-}$ mode in particular, and is at the $10 \%$ level at $2 \mathrm{GeV}, 30 \%$ between $1.5 \mathrm{GeV}$ and $3.0 \mathrm{GeV}, 100 \%$ above $3 \mathrm{GeV}$. The cross section is shown in Figure 6.

Figure 7 shows the measured cross sections for the $K_{S}^{0} K_{S}^{0} \pi^{+} \pi^{-}$and $K_{S}^{0} K_{S}^{0} K^{+} K^{-}$modes, systematically dominated by the background determination. The systematics errors are $5 \%$ at the peak region, $30 \%$ at $3 \mathrm{GeV}$, and range between $50 \%$ and $70 \%$ at higher energies. 

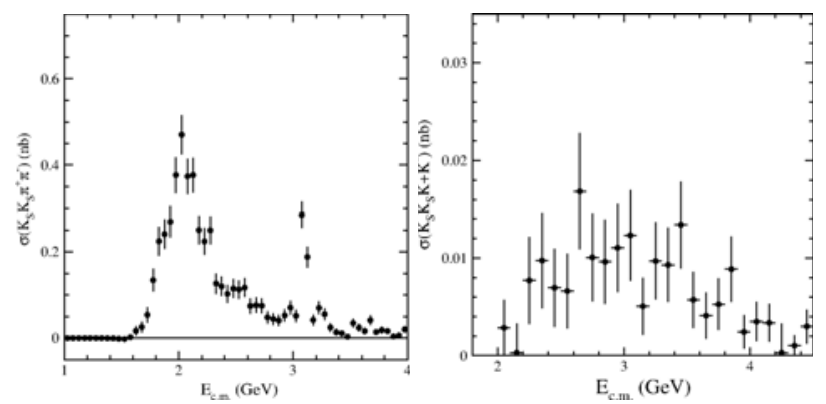

Figure 7. The $e^{+} e^{-} \rightarrow K_{S}^{0} K_{S}^{0} \pi^{+} \pi^{-}$and $K_{S}^{0} K_{S}^{0} K^{+} K^{-}$hadronic cross sections at BABAR. In the left plot the $J / \psi$ peak is visible. In the right plot the $J / \psi$ has been subtracted.

The measured branching fractions are $B\left(J / \psi \rightarrow K_{S}^{0} K_{S}^{0} \pi^{+} \pi^{-}\right)=(1.68 \pm 1.6 \pm 0.08) \times 10^{-3}$ and $B\left(J / \psi \rightarrow K_{S}^{0} K_{S}^{0} K^{+} K^{-}\right)=(0.42 \pm 0.08 \pm 0.02) \times 10^{-3}$. The $K_{S}^{0} K_{S}^{0} K^{+} K^{-}$branching ratio error is dominated by a $5 \%$ statistical uncertainty.

\section{Conclusions}

The hadronic cross sections for $a_{\mu}^{\text {had,VP }}$ dominate the uncertainties on $a_{\mu}^{\text {had }}$ and $a_{\mu}^{S M}$. Many recent results have improved the precision of the theoretical uncertainty on $a_{\mu}$, for example the BABAR $K^{+} K^{-}$ analysis has improved the estimate of $\delta a_{\mu}^{\text {had }}\left(K^{+} K^{-}\right)$by a factor 2.7. But discrepancies are evident for the most important channel in the region below $1.8 \mathrm{GeV}, \pi^{+} \pi^{-}$, and between the direct measurements and the SM calculations. In the near future new experiments, Fermilab g-2 (E989) [11] and J-PARC [12], will measure directly $a_{\mu}$ with a factor 4 overall improvement on the precision. And also the hadronic cross sections, involved in the SM calculations, should be better known from the CMD-3 [13] and BESIII [14] results and the future Belle2 [15] measurements.

\section{References}

[1] T. Kinoshita et al., Phys. Rev. Lett. 109, 111808 (2012).

[2] A. Czarmecki et al., Phys. Rev. D 67, 073006 (2003), Erratum ibid. D 73, 119901 (2006).

[3] S. J. Brodsky, E. De Rafael, Phys. Rev. 168, 1620-1622 (1968).

[4] G.W. Bennett et al., Phys. Rev. D 73, 072003 (2006).

[5] M. Davier et al., Eur. Phys. J. C 71, 1515 (2011).

[6] B. Aubert et al., The BABAR Collaboration, Nucl. Instrum. Meth. A729 615 (2013).

[7] B. Aubert et al., The BABAR Collaboration, Phys. Rev. Lett. 103, 231801 (2009).

[8] J. P. Lees et al., The BABAR Collaboration, Phys. Rev. D 86, 032013 (2012).

[9] J. P. Lees et al., The BABAR Collaboration, Phys. Rev. D 88, 032013 (2013).

[10] J. P. Lees et al., The BABAR Collaboration, Phys. Rev. D 89, 092002 (2014).

[11] G. Venanzoni et al., The E989 Collaboration, Nucl. Phys. Proc. Suppl. 225-227, 277-281 (2012).

[12] T. Mibe et al., the J-PARC $g$ - 2 Collaboration, Nucl. Phys. Proc. Suppl. 218, 242-246 (2011).

[13] G. V. Fedotovich et al., the CMD-3 Collaboration, Nucl. Phys. Proc. Suppl. 162, 332 (2006).

[14] M. Ablikim et al., The BESIII Collaboration, Nucl. Instrum. Meth. A 614 345-399, (2010).

[15] T. Abe et al., The Belle-II Collaboration, KEK Report 2010-1, arXiv:1011.0352 (2010). 